\title{
GIS-Based Remote Sensing Image Information Analysis of the South Atlantic Ecological Environment and Diversity of Deep-Sea Microorganism with Antifungal Effects
}

\author{
Jingjing $\mathrm{Xu}^{1,2}$ and Peisheng Yan $\mathbb{D}^{1,2}$ \\ ${ }^{1}$ School of Environment, Harbin Institute of Technology, Harbin, Heilongjiang 150000, China \\ ${ }^{2}$ School of Marine Science and Technology, Harbin Institute of Technology, Weihai, Shandong 264209, China \\ Correspondence should be addressed to Peisheng Yan; 21b929054@stu.hit.edu.cn
}

Received 20 November 2021; Revised 11 January 2022; Accepted 13 January 2022; Published 31 January 2022

Academic Editor: Miaochao Chen

Copyright (c) 2022 Jingjing Xu and Peisheng Yan. This is an open access article distributed under the Creative Commons Attribution License, which permits unrestricted use, distribution, and reproduction in any medium, provided the original work is properly cited.

This paper uses the GIS technology to collect location and geographic information of the South Atlantic ecological environment and then uses the remote sensing image technology to extract and analyse the collected information to provide a strong basis for maintaining a healthy marine ecological environment. Based on multitemporal remote sensing images, this paper constructs an ecological vulnerability evaluation model for marine by coupling several ecological indicators, analyses the status of marine ecological environment and its dynamic changes, explores the driving factors of its changes, further analyses the relationship between ecological vulnerability, land use changes, and landscape patterns, and proposes targeted ecological protection countermeasures for marine ecological environment. This paper analyses and studies the quality control mechanism of marine environment monitoring data, the construction of marine environment evaluation model, and the automation of evaluation and early warning response based on the application of GIS, with the support of marine environment evaluation and early warning business, to enhance the availability and integrity of marine environment monitoring data, improve the execution efficiency of marine environment evaluation business and early warning response, reduce the risk of marine environment emergencies, and realize the integration and intelligence of marine environment evaluation and early warning, to improve the level of Informa ionization and management of marine environment evaluation business in marine management departments. The operational watershed ecological environment monitoring service system built in this paper, which integrates GIS, RS, GPS, data warehouse, and other information technologies, has the advantages of high accuracy of information extraction, convenient data management, good portability, and strong operability, which can provide a scientific basis for the unified scheduling management of the marine environment, ecological environment evaluation, and sustainable development planning of the economy, and it is of great theoretical and practical significance and can be used in other watersheds. Meanwhile, the unique environment of the ocean has endowed the marine microorganism with affluent diversity, which can display a variety of bioactivities. In this study, thirty-two seawater and sediment samples were collected from different depths within the scope of $5 \mathrm{~m}$ to $3203 \mathrm{~m}$ at 18 sampling sites in the South Atlantic Ocean. 1017 culturable strains were obtained after isolation and identification. The tip culture method was used to screen strains with antifungal and antiaflatoxigenic activities. The tip culture results showed that a total of thirty-one marine strains were confirmed to have significant antagonism against aflatoxin production with an inhibition ratio exceeding $80 \%$, including 11 strains with a mycelial growth inhibition ratio exceeding $80 \%$ simultaneously. The variability of culturable bacterial distribution and diversity were studied by phylogenetic analysis of $16 \mathrm{~S}$ rRNA sequences. Phylogenetic analysis of $16 \mathrm{~S}$ rRNA sequences revealed a significant diversity of culturable strains. A total of 31 strains clustered into 13 genera: Marinobacter sp., Pseudomonas sp., Alteromonas sp., Idiomarina sp., Halomonas sp., Alcanivorax sp., Thalassospira sp., Jannaschia sp., Stakelama sp., Erythrobacter sp., Chengkuizengella sp., Bacillus sp., and Microbacterium sp. The 31 strains with antifungal and antiaflatoxigenic activities were dominated by populations of strains belonging to the genus Thalassospira (19.35\%). To the best of our knowledge, this is also the first report to analyse the diversity of culturable bacteria with antifungal and antiaflatoxigenic activities in the South Atlantic Ocean from three perspectives: different media, different sampling stations, and different sampling depths. 


\section{Introduction}

With the continuous improvement of science and technology and the rapid growth of population, people are changing the ecological environment in which they live at an unprecedented speed and scale, thus bringing about a series of ecological problems such as soil erosion, land sanding, and vegetation degradation, which pose a great threat to human production activities. Its changes have become a hot issue of concern to many experts and scholars at home and abroad. The strategy of "Ocean Power" promotes the development of the marine economy, which gradually makes the marine economy become a new growth engine of the national economy, and the protection of the marine environment is increasingly concerned by the public [1]. The sea area under China's authority is more than three million square kilometres, and the clear goal of the development of "Ocean Power" is to promote economic development from different perspectives, such as innovation in marine science and technology, formulation of national marine strategy, and scientific development of marine resources. And compared to land, the phenomenon in the ocean is fuzzy, and there is no obvious regional boundary. Therefore, ocean remote sensing data are more important to reflect the parameter attribute values under precise coordinates. The regional economic development of the country is gradually extending from a land-based economy to a comprehensive sea- and land-based economy [2]. Under the general trend of promoting the healthy and sustainable development of the sea economy, scientific planning of marine spatial structure, effective development of marine resources, high protection of the marine ecological environment, and the formulation of reasonable marine economic development strategies have become the focus of the work of coastal provinces.

Satellite remote sensing has the characteristics of wide coverage, a large amount of information, high timeliness, objectivity, multiresolution, and multitemporality, and the GIS technology has powerful spatial data management and spatial analysis capabilities. At present, new Earth observation technologies such as remote sensing and GIS have shown great potential for application in the field of risk prevention and control of major emergencies such as earthquakes, tsunamis, and floods and provide new means and methods for monitoring the marine ecological environment. The relevant departments in China have been using satellite remote sensing technology to carry out daily monitoring work in marine environmental monitoring for many years and have accumulated a wealth of monitoring data and information [3]. Under the current increasingly severe ecological environment situation, making full use of multisource data and multiple technical means to carry out marine risk zoning research is of great significance for environmental protection and safe travel and can provide a scientific basis for government decision-making, planning, and management. In this paper, we make full use of the remote sensing data products (MODIS, GLDAS, TRMM, STRM, HWSD, etc.), which are widely used in public platforms, and establish a platform for batch processing of data products from different data sources and different covariates. On the other hand, we combine the existing remote sensing and GIS data products to study the extraction methods of model parameters, such as vegetation cover, precipitation, surface albedo, soil moisture parameters, root depth, and other parameters [4].

The "digital ocean" was born with the concept of "digital Earth," which carries out real-time and continuous integrated data collection through satellites, remote sensing aircraft, maritime exploration vessels, seabed sensors, and so on and puts the basic information of the ocean into a "supercomputing system." In the "supercomputing system," the sea will be transformed into a virtual visual model of human development and protection of the ocean. The continuous development of the digital ocean will bring great changes to integrated ocean management services. With the continuous application of GIS in various marine work, its superiority is becoming increasingly obvious. GIS can store spatial data and its attribute data independently, to a certain extent, to achieve mutual operation; GIS system can intuitively interpret various graphic types of data and provide fast, accurate query and feedback. But with the continuous development and depth of various marine work, the real time, readability, accuracy, and other aspects of various types of marine data put forward higher requirements, and the application of GIS in the marine industry will also face new challenges. According to the characteristics of marine environmental analysis and evaluation of early warning work, GIS is better applied in various marine environmental evaluation and early warning works, and it is necessary to improve the support of GIS to marine environmental evaluation work.

\section{Status of Research}

GIS is a decision support system that began to develop rapidly in the $1960 \mathrm{~s}$. It is a computer system to collect, store, manage, analyse, describe, and apply data related to the spatial and geographical distribution of the whole or part of the Earth's surface. It consists of computer hardware and software systems, databases, and users, and intelligent GIS includes expert systems composed of knowledge and reasoning. GIS is an emerging edge discipline integrating geography, informatics, mapping science, computer science, spatial science, Earth science, and management science. It studies the combination of computer technology and spatial geographic distribution data through a series of spatial operations and analysis for geographic science, environmental science, and engineering design and even the development of the national economy, urban construction, and business operations to provide planning, management, and decision support information and to answer the relevant questions raised by users [5].

At present, ecological monitoring and assessment methods based on ecological significance are relatively mature, and most of such traditional ecological monitoring methods rely on fieldwork by ecologists and environmentalists, which ensures the accuracy of data but is timeconsuming, relatively expensive, and difficult to conduct ecological monitoring over a large area at a very narrow 
spatial scale [6]. It is also necessary to fine-correct the remote sensing image with reference to the 1:50000 current land use map of X city and unify the image into the same projection and coordinate system. In addition, the traditional ground measurement method only obtains limited information of physical parameters of ecological environment elements, which cannot reflect the spatial variability characteristics of such physical parameters information. Therefore, based on advanced and other technical means to carry out macroscopic, large-scale monitoring of dynamic changes in the ecological environment, it is especially necessary for the wide area of the Ta River basin to use remote sensing technology to carry out simultaneous and continuous monitoring of changes in the surface environment from the air over a large area, breaking through the limitations of environmental research from the ground in the past [7]. With the continuous development of remote sensing technology, a variety of satellite remote sensing images with different temporal and spatial resolutions have emerged, and the analysis of remote sensing data through the combination of RS and GIS has gradually moved from qualitative to quantitative. In 2002, the State Key Laboratory of Geographic Resources and Environmental Information System of the Academy of Sciences developed MaXplorer (Marine GIS explorer), the first marine GIS management system software with independent intellectual property rights in China, which filled the gap of this technology in China. In 2004, the scholars, led by Professor Chen, combined remote sensing technology with GIS technology to develop MAGIS, a marine atmospheric GIS platform software, which realized the seamless integration of marine atmospheric spatial and temporal analysis and visualization functions. The marine ecosystem service evaluation system developed based on the ArcGIS Engine platform has the functions of calculating the quality of ecosystem services and drawing and querying the spatial map of ecosystem services, making it easy for non-GIS professionals to make spatial maps of ecosystem services [8].

In summary, the development trend of ecological and environmental research at home and abroad in recent years can be summarized as the following characteristics: complexity and integration of research objects. The ecosystem is a natural-social-economic complex megasystem with the growth of population, economic development, and the enhancement of human ability to use resources. Ecological problems have become more complex and integrated, and research on the ecological environment has become more integrated and complex. Short-term scale and local problems of ecological environment research can no longer meet the needs of regional economic development and ecological coordination and must be carried out on a larger spatial scale and a long period cycle of ecosystem sustainability research quantification and modernization of research tools, ecological introduction to environmental research has been changed from qualitative to quantitative research represented by information technology, and so on [9]. Modern ecological environment research has gradually entered the stage of establishing regional sustainable ecosystems based on network-based long-term positioning monitoring and modern theoretical techniques as research tools. The establishment of digital ecological monitoring systems and the construction of digital watersheds at spatial scales larger than large watersheds has become an important development trend, and research objectives tend to be sustainable ecological and environmental management [10].

\section{GIS-Based Design of Marine Ecosystem}

3.1. GIS Marine Ecosystem Data Monitoring and Extraction. The marine ecological environment is very harsh, and accurate monitoring of information on the spatial and temporal evolution of different ecological factors throughout the region cannot be achieved by relying only on a single remote sensing platform. With the continuous development of remote sensing technology, especially the use of commercial satellites with multitemporal, multispectral, and multispatial resolutions, new technical means have been provided for the study of ecological and environmental information on a regional scale. The use of remote sensing image data to monitor the ecological environment has become a hot spot for research and application at home and abroad. At present, there are many satellite platforms for acquiring remote sensing data, and each has its advantages and disadvantages, such as TM/ETM + data of Landsat7 satellite with high spatial resolution, which can obtain surface coverage information with high accuracy, but its cycle is long, and its timeliness is poor; MODIS data with short operation cycle, large scanning range, and rich spectral information can extract rich surface feature parameters and thematic information, but the spatial resolution is very low; STOP5 and Quick Bird data have high spatial resolution and can obtain high-precision vegetation information, but the price is high [11]. Therefore, the bilinear interpolation method is used for sampling to control the error within the allowable range. Taking the corrected remote sensing image in 2003 as a reference, the 1987 remote sensing image was accurately corrected to achieve the precise fusion of the two phases of data. There are many monitoring contents and various data types, so the specific choice of satellite data and data extraction method needs to be considered according to the specific monitoring objectives, data contents, economic cost-effectiveness, and many other factors. In this paper, we take the sea area of the South Atlantic Ocean as an example to start a literature research survey.

The total source of freshwater recharge in the South Atlantic Ocean is atmospheric precipitation, which is poor and has few effective freshwater resources due to the fragmentation of the land area and the dispersion of the catchment. The South Atlantic Ocean has a year-round average of $1,223 \mathrm{~mm}$ and a total of 1,519 million cubic meters, the average multiyear runoff depth is $471.4 \mathrm{~mm}$, the total water resources are 586 million cubic meters, including a total of 145 million cubic meters of groundwater resources, salt flats, and seawater, and the flow produced on the land for aquaculture is equivalent to $25 \%$ of the total resources [12]. The land area of the archipelago's waters is $299,129.35 \mathrm{mu}$, accounting for $14.42 \%$ of the city's total land area. The fishing industry is well developed in X. Meanwhile, the salt 
industry is also traditional in the $\mathrm{X}$ archipelago. The $2015 \mathrm{X}$ City Marine Environment Bulletin shows that the environmental condition of seawater in the X archipelago's waters remains stable overall. The main exceedance factors of seawater in near-shore waters are inorganic nitrogen and reactive phosphate, with the best quality of seawater in August and the worst quality of seawater in March, and the water quality generally meets the use standard, as shown in Table 1.

Climate factors are an important element in the study of the ecological environment and an important indicator for the comprehensive evaluation of regional ecological environment status. For the information extraction of climate factors, determining and establishing indicator models and quantifying research data are the first problems that need to be solved. The data sources of climate factors come from the weather and climate bulletins updated every month, every quarter, and every year, and for the extraction process of climate factors, they are extracted based on annual precipitation, total evaporation, and annual sunshine duration data. Based on the spatial information of remote sensing and the meteorological data of weather and climate bulletin, the index model of the interrelationship of evaporation, precipitation, and annual sunshine radiation and temperature is established, which is used to analyse the climate pattern of the study area and to lay the foundation for the next step of calculating the comprehensive index of ecological and environmental conditions. The statistical analysis data of climate factors based on remote sensing satellite means that, compared with the conventional statistical means, the data of climate factor indicators based on remote sensing information has the characteristics of fast, accurate, laboursaving, and large and comprehensive information acquisition [13]. These indicators represent different physical connotations, so there are dimensional differences in these indicators. Therefore, before selecting the evaluation index, the evaluation index needs to be processed without dimension; that is, the influence of each evaluation index dimension is eliminated through mathematical transformation, and the original data of the evaluation index is standardized and standardized to avoid the impact of heterodimensional on the $\mathrm{X}$ islands. The evaluation results of the regional ecological environment have an impact. The temperature condition index (TCI) can be obtained by the method of remote sensing inversion. Tin represents the minimum value of the surface temperature of the study area in a certain period, and Tax represents the maximum value of the surface in a certain period.

GLDAS data were used to obtain the daily average temperature data of the Haihe River Basin for 2018, 2019, and 2020, and the temporal distribution characteristics of the temperature in the Haile River Basin were obtained through statistical analysis and batch processing procedures. The temporal variation pattern was analysed mainly from two aspects, interannual and monthly variations. From Figure 1, from the interannual variation, the average values of the basin-wide temperature in 2018, 2019, and 2020 were $6.3^{\circ} \mathrm{C}, 6.5^{\circ} \mathrm{C}$, and $6.8^{\circ} \mathrm{C}$, respectively, showing a slow upward trend. In terms of monthly variation, the highest
TABLE 1: Environmental quality of seawater in the South Atlantic Ocean (2017-2020).

\begin{tabular}{ccc}
\hline Year & One type & Second category \\
\hline 2017 & 0.483 & 0.638 \\
2018 & 0.785 & 0.932 \\
2019 & 0.971 & 0.953 \\
2020 & 0.704 & 0.775 \\
\hline
\end{tabular}

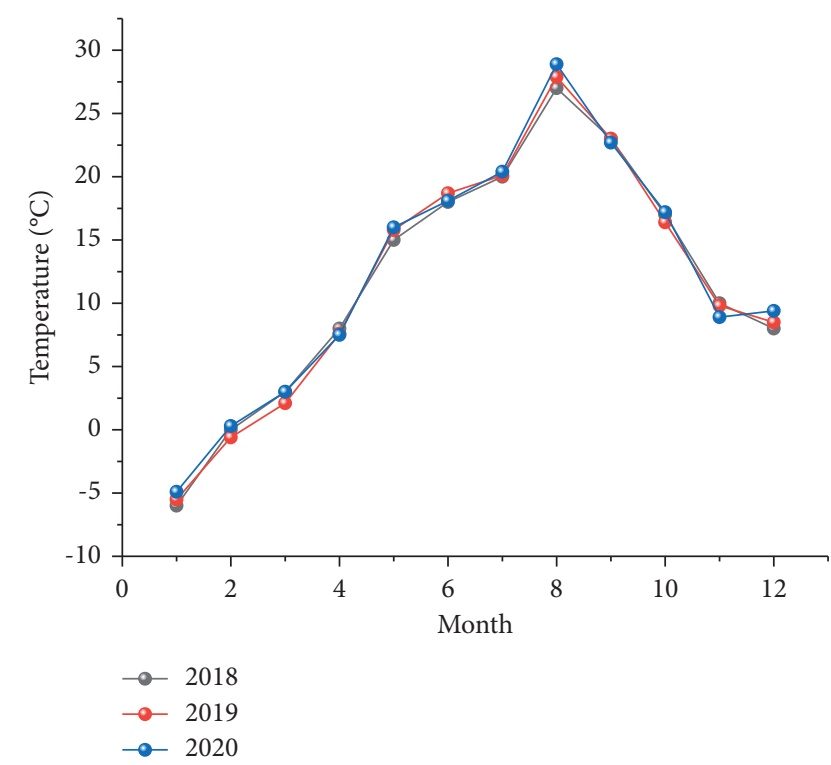

FIgURE 1: Monthly variation of sea temperature in the South Atlantic Ocean.

temperature values occurred in July. The spatial variation shows a decreasing trend from south to north from the plains to the mountains, which is analysed mainly due to the influence of altitude.

The survey and monitoring study of the distribution of water body resources, water quantity, water quality, and watershed area are important indicators for evaluating the status of the ecological environment. Information extraction methods for water bodies include the traditional field survey method, the statistical yearbook data query method, and the water-colour remote sensing technology, which has developed rapidly and has been increasingly used in recent years. Monitoring water body resources by satellite remote sensing has the characteristics of large coverage, wide monitoring range, short acquisition period, high data accuracy, and great data volume, which plays an important role in the monitoring and distribution density study of water bodies. The basic principle of water-colour remote sensing technology is that the water-colour sensor receives the solar radiation reflected by the water body and the scattering that occurred by the water body, which is formed by the visible and nearinfrared band absorption and special spectral characteristics; according to the difference of the spectral characteristics of the water body, water body index is enhanced, and the type of water body and the area of the water body can be analysed so that the water body information can be accurately extracted. Lakes, rivers, and reservoirs have the same 
composition of water bodies, all of which are formed by the accumulation of freshwater rainwater. The spectral curves of lakes, rivers, and reservoirs are the same, and they appear to be dark blue and black in the remote sensing images because of the weak reflective characteristics of freshwater and low spectral values. The shallow mudflats, which are composed of seawater, are highly reflective and have high spectral values due to the large amount of sediment carried by the Yangtze River into the sea, and they appear to be purple in the remote sensing images [14]. By comparing the spectral features, the information of the water bodies can be extracted accurately. The extraction of surface runoff and total groundwater resources in the study area can be studied by using the water body equilibrium relationship, in addition to consulting the statistical yearbook. According to the principle of water body circulation, where $R$ is the total precipitation in the study area, Rs is the surface runoff, $R$ is the total groundwater resources, and $E$ is the total evaporation calculated in the climate factor, the formula is as follows:

$$
R=R_{s}-R_{g}+E^{2}
$$

3.2. Remote Sensing Image Data Analysis. The raw remote sensing images are affected and interfered with by various factors such as the curvature of the Earth, atmospheric refraction, sunlight irradiation, and the scanning angle of the remote sensor during the imaging process, resulting in poor imaging quality of the target object and inaccurate reflection of its electromagnetic energy, so the raw remote sensing images need to be preprocessed to reduce the influencing factors in the imaging before being used for analysis. The computer classification of remote sensing images is mainly based on the similarity of the grey values of the pixels of the image. The similarity between the pixels is measured according to the distance and correlation coefficient. The greater the similarity is, the closer the attributes of the pixels are and the more likely it is to be classified into one category. The raw remote sensing data were preprocessed using ENVI5.2 software. The selected HMS-2 remote sensing satellite image is panchromatic data. According to the HMS2 preprocessing flowchart, the preprocessing steps for the raw image should be data opening $\longrightarrow$ orthorectification $\longrightarrow$ autoalignment (optional) $\longrightarrow$ image fusion $\longrightarrow$ fast atmospheric correction, as shown in Figure 2.

In this section, the image tile construction technique is used to realize the unified indexing of multisource remote sensing data. Image tile data is generated by resampling or cutting the original remote sensing image according to certain rules, its file is smaller, and the number of files is larger compared with the original remote sensing image, so it has more advantages than the original image data in terms of data reading and writing speed, processing efficiency, and local range analysis. The current image tile construction methods mainly include the image pyramid construction technique and the image chunk construction technique. Both these two construction methods effectively solve the problem of analysis and processing difficulties caused by the excessive data volume in the application of high-resolution remote sensing images. However, considering that the resolution of different sensors is not consistent, in the scenario of using multisource remote sensing data, it still needs to be unified by resampling before the calculation and analysis. For remote sensing data with different resolutions, the largest tiles of the lower resolution image can be calculated directly with the corresponding tiles of the higher resolution image without resampling. Compared with the image chunking construction technique, the image pyramid technique achieves unified management of multiresolution remote sensing data by sacrificing the storage space of data to a certain extent. Therefore, we use the image pyramid construction technique to preprocess the original multisource multiresolution remote sensing data and finally perform the image tile generation through a quadratic tree with a cascading structure.

$$
p=\frac{\rho_{I}^{2}+\rho_{K R}^{2}}{\left(\rho_{I}-\rho_{K R}\right)\left(\rho_{I}+\rho_{K R}\right)} .
$$

Usually, the image pyramid structure starts from the highest level where every four neighbouring pixels are resampled to generate a new pixel and so on until the end of the 0th level. And for better image grayscale effect after resampling, its resampling method prefers interpolation methods such as bilinear interpolation or cubic convolution method that considers multiple neighbouring pixels of a pixel without considering the efficiency. However, the image pyramids in this paper are more computationally oriented; that is, the resampling of the remaining layer tiles needs to be implemented for the highest layer tiles of the lower resolution images to be computed directly with the corresponding layer tiles of the higher resolution images. The ocean is fuzzy compared with the land, and there is no obvious regional boundary, so the ocean remote sensing data is more important to reflect the parameter attribute values in precise coordinates. The above two points are combined, and the tiles of the remaining layers are also resampled by the nearest neighbour method. That is, the pixel value of a certain grid point after resampling represents only the parameter attribute value at that latitude and longitude. This representation is also more reasonable considering that geostationary satellites such as GOCI will mark the latitude and longitude values of each pixel point in detail in the remote sensing raw data file. In addition, since the highestlevel tiles have been resampled by the nearest neighbour method, that is, the pixel values of all grid points of the highest-level tiles represent the parameter attribute values at the latitude and longitude of their grid points, the remaining level tiles only need to be resampled at pixel intervals from the upper-level tiles. The image pyramid tile set constructed by the above way for multisource remote sensing data can realize the direct calculation of the same layer tiles of different resolution remote sensing data, that is, the unified organization and management of multisource multiresolution remote sensing data, as shown in Figure 3.

In this chapter, according to the characteristics of large area and large data volume of remote sensing data and the 

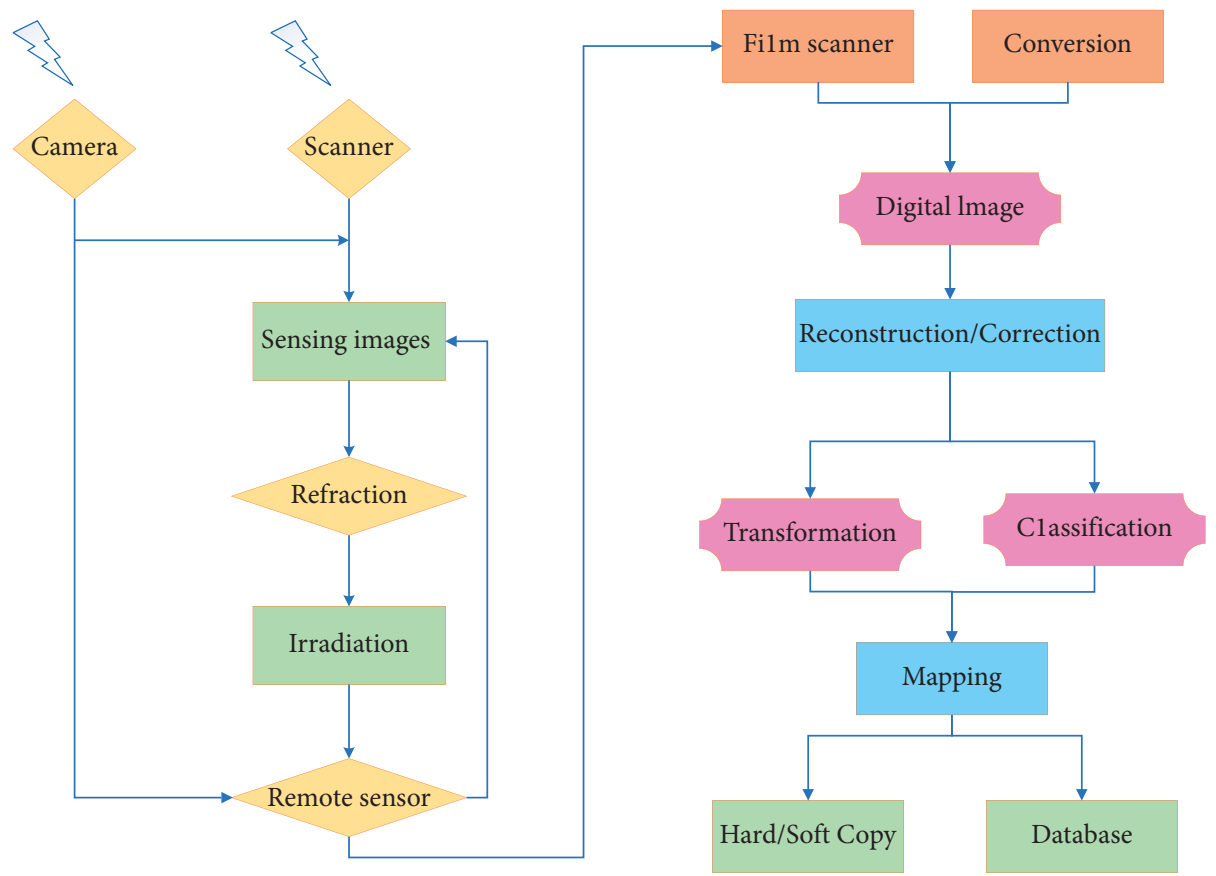

FIGURE 2: Preprocessing process of remote sensing data of HMS-2.

resulting difficult problem of processing and utilizing long time-series multisource remote sensing data, we choose to adopt the image pyramid approach to unify the processing of multisource multiresolution remote sensing images by constructing a unified index of them in the form of sameresolution hierarchical tiles and form a Patio-temporal tile matrix based on this for the inversion of time-series data and extraction. The biggest advantage of the pyramid is that only the data of the specified resolution and region are retrieved, which can greatly improve the computational speed of raster data.

To obtain the spatial distribution and size of wetlands, remote sensing images are required to ensure the correctness of the location, while the original remote sensing images are direct descriptions of the irregular Earth surface, and there are certain deviations between the location of geographical entities represented by remote sensing images and the location in reality because of Earth curvature, atmospheric refraction, and sensor errors. And for red tide events that occur in different time and space, the method of setting multiple target analysis points can avoid the difference of chlorophyll-a concentration in different time and space from forming abnormal detection standards and eventually leading to misjudgement. Although the remote sensing data used in this paper have been atmospherically corrected and coarsely geometrically corrected by the satellite ground receiving station, that is, the influence of atmospheric radiation on features and the geometrical distortion caused by the satellite itself is eliminated, they still cannot meet the research needs, and the remote sensing images must be finely corrected and unified into the same projection and coordinate system concerning the $1: 50,000 \mathrm{X}$ city land use status map. The commonly used fine correction methods are the polynomial correction method and the common line equation correction method [15]. The polynomial correction method avoids the spatial geometric process of remote sensing imaging and simulates the remote sensing image itself mathematically, which is widely used due to its simple principle and easy operation, but it cannot correct the error caused by the change of terrain and is not suitable for areas with large topographic relief. The common equation correction method is based on the strict mathematical transformation relationship between image coordinates and ground coordinates and is a direct description of spatial geometry, which can correct the errors caused by terrain changes and is more suitable for mountainous areas with large terrain difficulties.

$$
K=\left[\begin{array}{cccc}
a_{1} & a_{2} & \ldots & a_{n} \\
a_{11} & a_{12} & \ldots & a_{1 n} \\
\ldots & \ldots & \ldots & \ldots \\
a_{n 1} & a_{n 2} & a_{n 3} & a_{n n}
\end{array}\right] .
$$

After calibration, the data were also resampled to check the calibration results. By resampling, we mean the process of reassigning values to image elements according to certain rules. The three main methods are the nearest distance method, cubic convolutional interpolation, and bilinear interpolation. The nearest distance method assigns the nearest image element to the new image element, which is fast, but may cause incoherence of some surface forms. The bilinear interpolation method is the most used and uses the image element values of four neighbouring points for interpolation. The triple convolution method is slow in processing as it must consider 16 surrounding image elements. The bilinear interpolation method is moderate in computation and accuracy among the three 


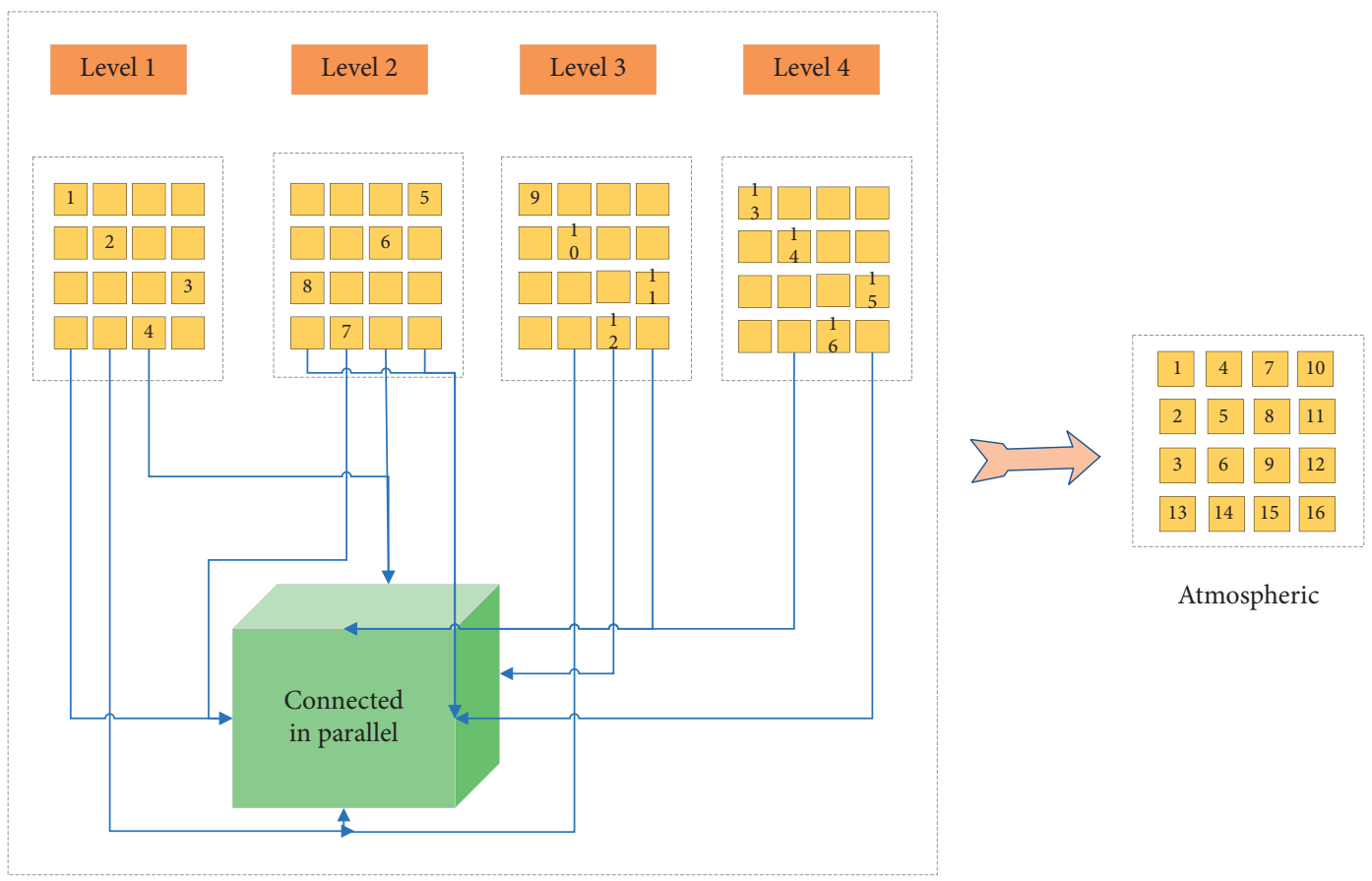

FIgURE 3: Schematic diagram of grid reorganization.

methods. To avoid the disadvantages of incoherent features of the nearest distance method and the slow processing speed of the three convolution methods, the bilinear interpolation method is used to sample and control the error within the allowable range. Then, the 2003 remote sensing image completed by the correction is used as the reference, and the 1987 remote sensing image is refined and corrected to achieve the accurate fusion of the two phases of data.

3.3. Sources of Marine Strains. The seawater and sediment samples used in this study were collected at different stations in the South Atlantic Ocean during the No. 26 ocean Cruise by R/V Dayang 1 in 2012. Seawater samples from different depths $(5-2,700 \mathrm{~m})$ of the South Atlantic Ocean were collected from 3 sites [16]. Sediment samples from different depths $(451-3,203 \mathrm{~m})$ of the South Atlantic Ocean were collected from 15 sites, and the details of sampling sites and depths are presented in Table 2. Once retrieved, samples were immediately stored at $4^{\circ} \mathrm{C}$ and microorganisms were isolated in the onboard laboratory.

3.4. Selection of Antibacterial Indicator Bacteria. The indicator strain used in this experiment was an Aspergillus parasiticus mutant strain NFRI-95, which did not produce aflatoxin but accumulated norsolorinic acid (NA), the first stable precursor in the synthetic pathway of aflatoxin. NA was bright red and visible to human eyes. The indicator strain NFRI-95 in this experiment was provided by Dr. Yabe from Japan.
TABle 2: Sampling stations, dive date, depths, and sample description.

\begin{tabular}{cccc}
\hline Station & Dive date & Depth $(\mathrm{m})$ & Sample description \\
\hline TVG01 & 20120725 & 3203 & Light yellow slime \\
TVG02 & 20120726 & 3141 & Reddish brown slime \\
TVG03 & 20120726 & 3142 & Black basalt clasts \\
TVG04 & 20120727 & 3149 & Red-brown fine sand \\
TVG05 & 20120727 & 3125 & Calcium foraminifera clay \\
TVG06 & 20120727 & 3059 & Tawny lumps \\
TVG08 & 20120728 & 3051 & Detrital clay \\
TVG09 & 20120729 & 3073 & Reddish brown slime \\
TVG10 & 20120802 & 2770 & Sulfide chimney wall \\
TVG12 & 20120803 & 2721 & Polymetallic sulfide \\
TVG13 & 20120806 & 2730 & Basaltic lava \\
TVG14 & 20120807 & 2796 & Light yellow calcium slime \\
TVG15 & 20120808 & 1523 & Earthy yellow ooze \\
TVG16 & 20120808 & 1598 & Fine sand calcium slime \\
TVG21 & 20120814 & 451 & Surface calcareous deposits \\
\hline
\end{tabular}

3.5. Media. The eleven cultivation media and their compositions are as follows:

(1) $2216 \mathrm{E}$ medium: $1 \mathrm{~g}$ yeast extract, $0.1 \mathrm{~g}$ iron phosphate, $5 \mathrm{~g}$ peptone, $1000 \mathrm{~mL}$ seawater, and $\mathrm{pH} 8.0$

(2) R2A medium: $0.3 \mathrm{~g}$ dipotassium hydrogen phosphate, $0.5 \mathrm{~g}$ yeast extract, $0.5 \mathrm{~g}$ peptone, $0.15 \mathrm{~g}$ casein hydrolysate, $0.15 \mathrm{~g}$ glucose, $0.3 \mathrm{~g}$ sodium pyruvate, $0.05 \mathrm{~g}$ magnesium phosphate, $0.5 \mathrm{~g}$ soluble starch, and $1000 \mathrm{~mL}$ seawater

(3) M2 medium: $0.5 \mathrm{~g}$ yeast powder, $0.5 \mathrm{~g}$ beef extract, $0.5 \mathrm{~g}$ soluble starch, $0.5 \mathrm{~g}$ sucrose, $5 \mathrm{~g}$ sodium acetate, $0.5 \mathrm{~g}$ peptone, $0.5 \mathrm{~g}$ glucose, $0.05 \mathrm{~g}$ sodium citrate, 
$1.0 \mathrm{~g}$ ammonium nitrate, $0.05 \mathrm{~g}$ potassium sodium tartrate, $0.05 \mathrm{~g}$ malic acid, $0.2 \mathrm{~g}$ ammonium chloride, $1000 \mathrm{~mL}$ seawater, and $\mathrm{pH} 7.5-7.6$

(4) F medium: $1 \mathrm{~g}$ glucose, $4 \mathrm{~g}$ beef extract, $4 \mathrm{~g}$ peptone, $1 \mathrm{~g}$ yeast extract, $1000 \mathrm{~mL}$ seawater, and $\mathrm{pH} 7.0$

(5) $\mathrm{G}$ medium: $10 \mathrm{~g}$ peptone, $8 \mathrm{~g} \mathrm{Na} \mathrm{Na}_{2} \mathrm{HPO}_{4}, 2 \mathrm{~g}$ $\mathrm{K}_{2} \mathrm{HPO}_{4}, 1000 \mathrm{~mL}$ seawater, and $\mathrm{pH} 7.1-7.3$

(6) Starch casein medium: $10 \mathrm{~g}$ soluble starch, $4 \mathrm{~g}$ yeast extract powder, $2 \mathrm{~g}$ casein, and $1000 \mathrm{~mL}$ seawater

(7) Gause No. 1 medium: $20 \mathrm{~g}$ soluble starch, $1 \mathrm{~g}$ potassium nitrate, $0.5 \mathrm{~g}$ dipotassium hydrogen phosphate, $0.5 \mathrm{~g}$ magnesium sulfate heptahydrate, $0.01 \mathrm{~g}$ ferrous sulfate heptahydrate, $0.5 \mathrm{~g}$ sodium chloride, and $1000 \mathrm{~mL}$ seawater

(8) Glucose-asparagine medium: $10 \mathrm{~g}$ glucose, $0.5 \mathrm{~g}$ asparagine, $0.5 \mathrm{~g}$ dipotassium hydrogen phosphate, and $1000 \mathrm{~mL}$ seawater

(9) W1 medium: $9.3690 \mathrm{~g}$ yeast powder, $2.0820 \mathrm{~g}$ malt extract, $13.8060 \mathrm{~g}$ glucose, $\mathrm{pH}$ 6.2, and $1000 \mathrm{~mL}$ tap water

(10) GY medium: $20 \mathrm{~g}$ glucose, $5 \mathrm{~g}$ yeast powder, and $1000 \mathrm{~mL}$ seawater

3.6. Cultivation and Measurement of Antifungal/Antiaflatoxigenic Activity. The overnight liquid seed culture of isolated strains was inoculated into $50 \mathrm{ml}$ Erlenmeyer flasks containing $20 \mathrm{ml}$ corresponding liquid media, respectively. The inoculation size was $1 \%(\mathrm{v} / \mathrm{v})$. The cultures were cultivated at $28^{\circ} \mathrm{C}$ with shaking at $120 \mathrm{rpm}$ for 6 days. Each experiment was carried out in three replicates. After the cultivation, the cell suspension was centrifuged at $8,000 \times \mathrm{g}$ for $20 \mathrm{~min}$ at room temperature. The supernatant was supplemented with GY $(2 \%$ glucose and $0.5 \%$ yeast extract) to compensate for the consumption of nutrients by bacterial growth, and the $\mathrm{pH}$ of the medium was adjusted to approximately 6.0. After filter sterilization with a $0.22 \mu \mathrm{m}$ pore-size Millipore membrane, the resulting solution was used as a cell-free supernatant for antifungal and antiaflatoxigenic bioassays. For determination of the antifungal activity, the tip culture method was used, using A. parasiticus mutant strain NFRI-95 as an indicator in these bioassays $[17,18]$. To test the antiaflatoxigenic activity (inhibition of red pigment production), norsolorinic acid in the mycelia was extracted with a solution containing $1 \mathrm{~mol} / \mathrm{L}$ $\mathrm{NaOH}$ and methanol $(1: 9, \mathrm{v} / \mathrm{v})$. Then, $\mathrm{OD}_{560 \mathrm{~nm}}$ of the extract was measured. Uncultivated media supplemented with GY and inoculated with $A$. parasiticus NFRI-95 were used as controls for tip culture. The inhibition ratio of mycelial growth and aflatoxin was calculated according to the following formulas:

Mycelial growth inhibition ratio $(\%)=(X 2-X 1) /$ $X 2 \times 100 \%$ with $\mathrm{X} 2$ : fresh weight of the mycelia in the control tip and $X 1$ : fresh weight of the mycelia in the experiment tip

Aflatoxin inhibition ratio $(\%)=(Y 2-Y 1) / Y 2 \times 100 \%$ with $Y 2: \mathrm{OD}_{560 \mathrm{~nm}}$ of the control tip and $Y 1$ : OD560nm of the experiment tip
3.7. 16SrRNA Sequencing and Phylogenetic Analysis. Genomic DNA from the microorganisms was extracted using a bacterial genomic DNA FastPrep Extraction Kit (SaiBaiSheng Gene, Shanghai), according to the manufacturer's protocol.

Polymerase chain reaction (PCR) amplification of the nearly full-length $16 \mathrm{~S}$ rRNA gene was performed using the universal primers 16SF $\left(5^{\prime}\right.$-AGAGTTTGATCCTGGCTCAG) and 16SR (5'-ACGGCTACCTTGTTACGACT). PCR was performed using the extracted highly purified genomic DNA as a template under the following conditions: $94^{\circ} \mathrm{C}$ for $5 \mathrm{~min}$, followed by $94^{\circ} \mathrm{C}$ for $40 \mathrm{~s}, 55^{\circ} \mathrm{C}$ for $40 \mathrm{~s}$, and $72^{\circ} \mathrm{C}$ for $1 \mathrm{~min}$ for 32 cycles with a final $10 \mathrm{~min}$ extension at $72^{\circ} \mathrm{C}$. The primer for PCR sequencing was $\mathrm{P} 300$ (5'-CCAGACTCCTACGGGAGGCAGC).

Similarity searches of all the sequences were performed in the GenBank database using BLASTn and the EzBiocloud database using EzTaxon server 2.1. The sequence alignments were performed using CLUSTAL_X, and the phylogenetic trees were constructed from evolutionary distances using the neighbour-joining method of the MEGA 5 program package.

\section{Results and Analysis}

4.1. GIS-Based Performance Results for Marine Ecosystems. Based on the factors affecting the evaluation of the regional ecological environment of Zhoushan Islands based on RS and GIS, the first and most important step to evaluate the ecological environment of Zhoushan Islands is to establish a suitable indicator system. The establishment of the indicator system is the most basic prerequisite for quantitative evaluation of the regional ecological environment of the Zhoushan Islands and is also directly related to the accuracy and science of the ecological environment evaluation of the study area. In the second chapter, the natural, economic, and social profiles of Zhoushan Islands are introduced in detail, and the actual situation of ecological environment in the study area is analysed in combination with the profiles, while reference is also made to the selection methods of domestic and foreign scholars for the indicator system, following the principles of operability, representativeness, and practicality, and a comprehensive indicator system is selected, the multisystem evaluation indicator system, which is different from the single type of regional indicator system [19]. Abundant surface feature parameters and thematic information can be extracted, but the spatial resolution is very low; STOP5 and QuickBird data have very high spatial resolution and can obtain high-precision vegetation information, but the price is relatively high. In the comprehensive multi-indicator evaluation, two basic variables are involved, one is the actual value of each evaluation indicator, and the other is the evaluation value of each indicator, and the different physical connotations are represented by these indicators, so there are differences in the scale of these indicators. Therefore, before selecting evaluation indicators, it is necessary to carry out dimensionless processing of evaluation indicators, that is, to eliminate the influence of each evaluation indicator's dimension through 
mathematical transformation, so that the original data of evaluation indicators can be standardized and specified, thus avoiding the influence of heterogeneity on the evaluation results of the regional ecological environment of South Atlantic Ocean. There are three main methods of dimensionless processing of evaluation indexes: linear dimensionless method, folded dimensionless method, and curved dimensionless method; because of the multi-indicator comprehensive evaluation index system adopted, the folded dimensionless method, which is most suitable to be applied to the multi-indicator comprehensive evaluation, is chosen, which is simpler and more certain than the curved dimensionless method and is also more in line with the actual multisystem evaluation index system requirements. Changes in evaluation indicators in different regions of the South Atlantic Ocean have different effects on the comprehensive evaluation results. The equation for the treatment of evaluation indicators by the curvilinear dimensionless method is as follows:

$$
y_{t}=y_{m}+\frac{x_{i}^{2}+x_{m}^{2}}{\min \left\{x_{i}\right\}}\left(1+y_{m}\right)
$$

$X$ is in the southern subtropical region and has a southern subtropical maritime monsoon climate. The average year-round temperature ranges from $19^{\circ} \mathrm{C}$ to $19.9^{\circ} \mathrm{C}$. The highest temperature of the South Atlantic Ocean is from July to August, generally $32^{\circ} \mathrm{C} \sim 35^{\circ} \mathrm{C}$, and the lowest temperature is in February, generally $2^{\circ} \mathrm{C} \sim 4^{\circ} \mathrm{C}$. The annual precipitation of the South Atlantic Ocean is $900 \sim 1200 \mathrm{~mm}$, decreasing from northwest to southeast. The most precipitation is from March to September, the intensity of water is the greatest in June, followed by July to September, and the least is in October to February. Pingtung Island is under the influence of monsoons all year round, with mostly southerly winds in summer and northerly winds in spring, autumn, and winter. Since the island is in the Taiwan Strait and adjacent to the Pacific Ocean, it is vulnerable to typhoons and storm surges throughout the year. Typhoons are accompanied by heavy rains, and September is the month when heavy rains occur. Therefore, it is vulnerable to typhoons and storm surges throughout the year. Typhoon is the disaster that affects Pingtung Island the most, especially the typhoon generated in the northwest Pacific Ocean, often these typhoons will land from the front, the wind is strong, and at the same time, the rainfall is high, causing a very serious impact on Pingtung Island. Some of the typhoons with great impact are Fetyan, Trazitiline, Longwing, and Morakot. Precipitation on South Atlantic Ocean for different months is shown in Figure 4.

\section{Results of Remote Sensing Image Data Processing}

Similar features on remote sensing images have the same spectral characteristics and spatial distribution characteristics under the same physical conditions, while different features have different spectral characteristics and spatial distribution characteristics, and these feature differences are

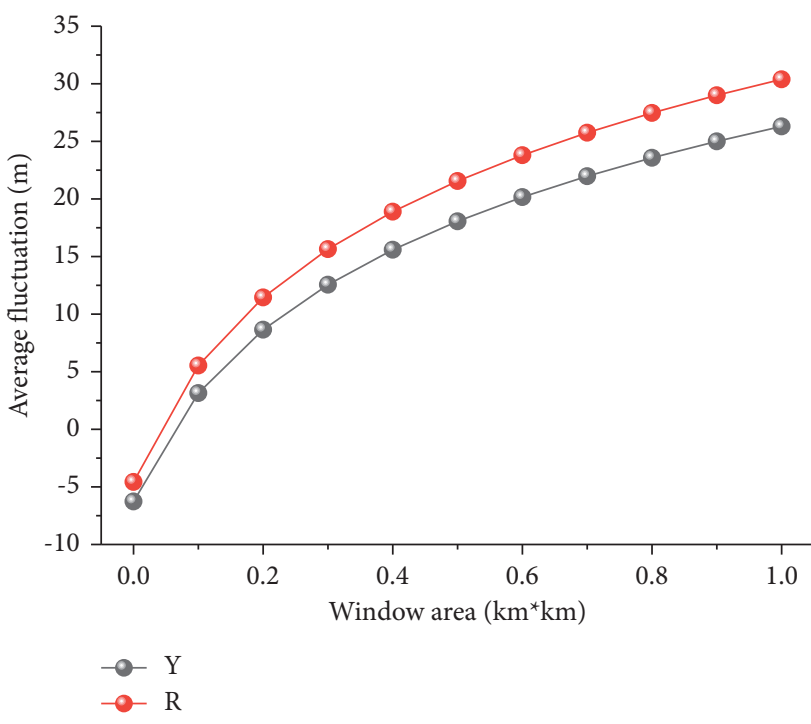

FIgUre 4: Precipitation on South Atlantic Ocean for different months.

the physical basis for distinguishing different features in the images. In the days when computer technology was not yet developed, the classification of remote sensing images was mainly through human visual interpretation. With the popularization and development of computers, the classification of remote sensing images began to enter the digital era. The current stage of remote sensing image classification is to identify the spectral characteristics and spatial distribution characteristics of different features with the help of a computer and then realize the extraction of the features [20]. The computer classification of remote sensing images is mainly based on the similarity of image elements' grayscale values, the similarity between the elements is measured according to the distance and correlation coefficient, and the greater the similarity, which indicates that the properties of the elements are more similar, the greater the possibility of being classified into one category. There are three main categories of classification methods, which are supervised classification, unsupervised classification, and decision tree classification.

$$
\begin{aligned}
S_{n}= & \lim _{m \longrightarrow \infty} \sum_{t=2}^{m-i}\left(x_{1}-x_{m 1}\right)^{3} \\
& -2 \lim _{m \longrightarrow \infty} \sum_{t=m}^{m}\left(x_{1}-x_{m 2}\right)\left(x_{1}-x_{m 1}\right) \\
& +\lim _{m \longrightarrow \infty} \sum_{t=2}^{m-i}\left(x_{1}-x_{m 2}\right)^{3} .
\end{aligned}
$$

To improve the speed and accuracy of graph vectorization, a special engineering legend board has been established, through which the specific parameters of the current points, lines, and surfaces can be obtained during vectorization, without having to reset them each time the graph is entered. In the MaPGis 6.6 graphics editor main window file management platform, click New Project Legend, the legend editing dialog box appears, select the district type legend, in 
the legend information in the name field, enter the marsh wetland, in the legend parameters, enter the specific parameters of the marsh wetland, fill colour select 935, fill pattern select 157, pattern width and pattern height are selected 500, pattern colour and layer are selected $\mathrm{O}$, parameters are set, and click the Add button to add the new district symbol just now; follow this method to create a river wetland, reservoir wetland, salt flats, and other district type symbols. The data sources of climate factors come from the weather and climate bulletins updated every month, every season, and every year. The extraction process of climate factors is based on the data of annual precipitation, total evaporation, and annual sunshine time. The point legend and line legend were created in the same way as the zone legend. After creating the legends required for this paper, click the OK button at the top left of the dialog box to close the engineering legend editing dialog box. Right-click in the file management platform, click Associate Legend File, and find and open the legend board file you just created according to the path prompt dialog box for vectorization [16]. The parameters of the drawn graph and the parameters of the legend on the legend board by mouse click are the same. After drawing a kind of landscape, click the legend of the landscape you want to draw with the mouse, and the parameters of the graph change to the parameters of the selected legend, as shown in Figure 5.

When classifying land use from remote sensing imagery, whether supervised, unsupervised, or decision tree classification is used, the basic principle is to perform cluster analysis based on the spectral characteristics exhibited by various features in the imagery. This leads to a certain degree of blindness in the classification process. The classification results obtained will have small areas of spots, such as isolated points, holes, breaks, and burrs, which will image the quality and accuracy of the resulting map. Therefore, postclassification processing of the image land use classification results is needed to remove the defects in the images. In this paper, soil salinization extraction is done in the same way as land desertification by using a hierarchical classification method, firstly, by image calibration, setting empirical thresholds, and calculating, the calibration results are divided into vegetation and nonvegetation classes, and then the information is extracted step by step.

$$
y=\sqrt{\left(x-x^{\prime}\right)^{2}}-\sqrt{\left(y^{\prime}-y\right)^{3}} .
$$

The nonvegetation class performs a spike cap transformation on the nonvegetation data to classify features into waterbody and nonwaterbody classes based on their moisture information, which is extracted as follows.

Nonaqueous category: according to the spectral curve, it is known that the water body and the residential land area are in the strong absorption and low reflection region in the band, and the two can be extracted by setting the threshold value.

Water bodies: masking water bodies and settlement information, calculating vegetation indices for calibration results, and setting thresholds to extract nonsalinized and salinized classes. The nonsalinization class is extracted by setting the empirical Min value, and the salinization class is extracted by density segmentation technique using the vegetation index, while the extraction of soil salinization is completed by supervised classification method utilizing mask processing, principal component transformation, band operation, minimum distance classification, and sample area selection in interest, as shown in Figure 6.

The regional ecological condition and grade distribution of the South Atlantic Ocean are closely related to the natural environment and human activities in the region, and whether the ecological environment becomes better or worse, the magnitude of change is not large, and the change is not a sudden and violent process, but a result of gradual accumulation, so in the process of protecting and restoring the ecological environment, building an ecological civilization, and creating a green marine ecological environment, attention should also be paid to the gradual and comprehensive development.

In summary, the proposed framework for the detection of environmental anomalies in marine red tide from long time-series multisource remote sensing data is effective for the identification of red tide hazards. Specifically, the Patiotemporal tile model proposed in this paper unifies the multisource multiresolution GOCI, MODIS, G1SST, and OCCCI data and greatly facilitates the extraction of timeseries data, and the Patio-temporal feature body structure in this model significantly improves the efficiency of range query [17]. The basic principle of the water-colour remote sensing technology is that the water-colour sensor receives the solar radiation reflected by the water body and the scattering of the water body, which is absorbed by the visible light and near-infrared bands to form special spectral characteristics. According to the difference in the spectral characteristics of the water body, the water body index is enhanced and can be analysed. The type of water body and the area of the water area can accurately extract the water body information. At the same time, the proposed method for detecting environmental anomalies in marine red tide is based on the chlorophyll-a concentration, which can dynamically screen out the real anomalies; that is, based on the multiyear time-series analysis, instead of assessing the chlorophyll-a concentration at a single moment, a dynamic threshold is used for screening based on the prediction error between the predicted and real values, avoiding the high chlorophyll-a concentration at a single moment for the assessment of the real anomalies. This avoids the pseudoanomaly caused by high chlorophyll-a concentration at a single moment. In addition, for red tide events occurring in different times and spaces, multiple target analysis points can be set to avoid the difference in chlorophyll-a concentration between different times and spaces, which may lead to different criteria for anomaly detection and eventually lead to misjudgement, as shown in Figure 7.

Strains with aflatoxin inhibition ratio and mycelial growth inhibition ratio exceeding $80 \%$ 


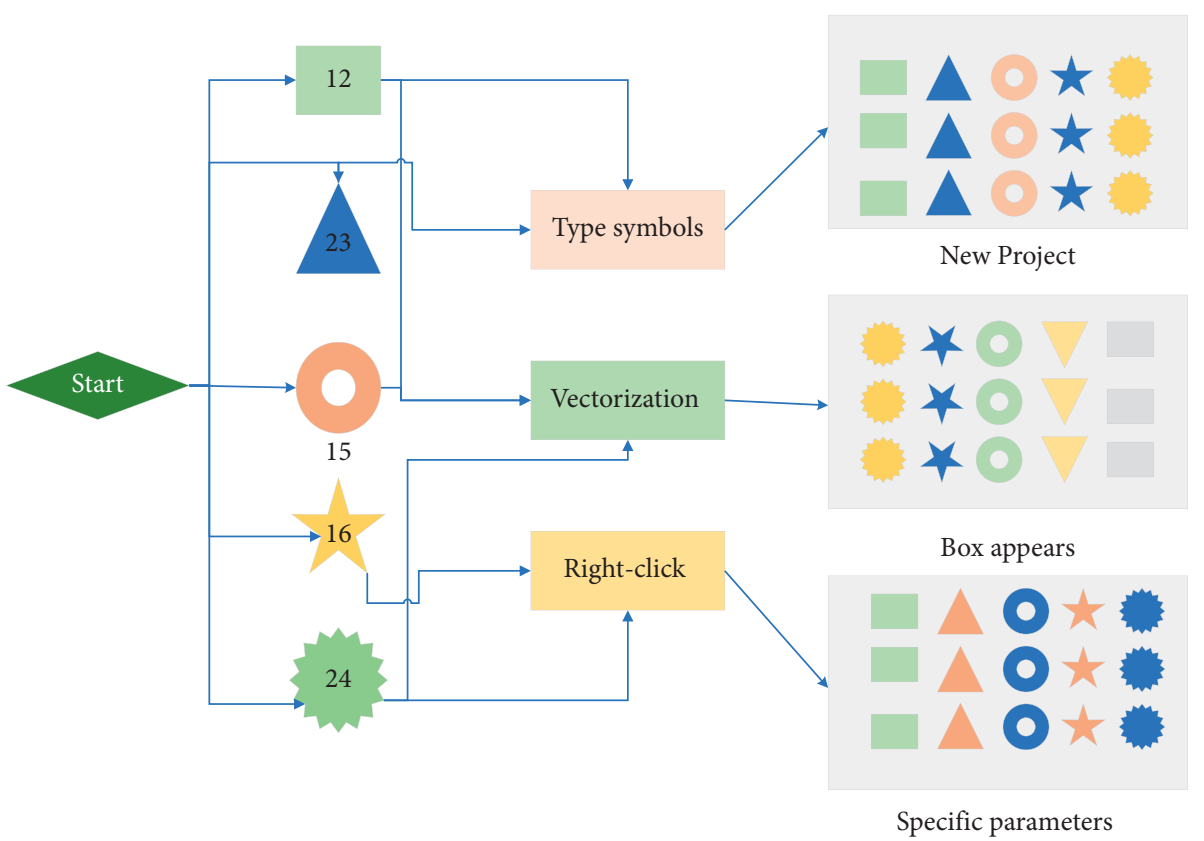

FIgURE 5: Legend edit dialog.

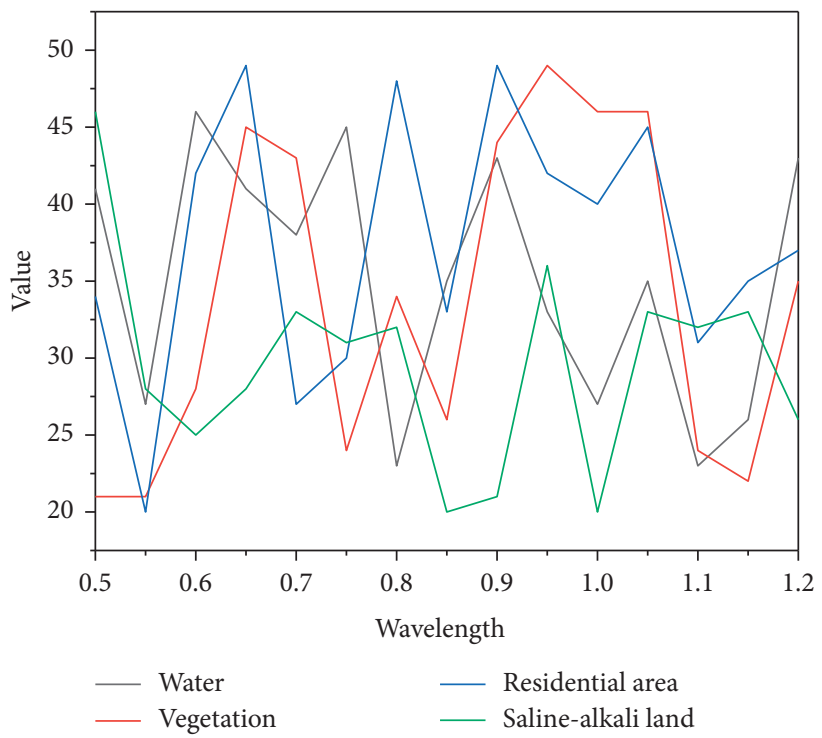

Figure 6: Spectral profile of the feature.

In this experiment, a total of 1017 strains were isolated and identified, including 218 seawater strains and 799 strains isolated from deep-sea sediments. All of them took part in screening by tip culture method. The results showed that there were 31 strains against aflatoxin production with an inhibition ratio exceeding $80 \%$, including 11 strains with a mycelial growth inhibition ratio exceeding $80 \%$ simultaneously (Tables 3 and 4). These 31 strains contained 4 strains collected from seawater and 27 strains collected from sediment.
5.1. Phylogenetic Analysis of Culturable Strains with High Inhibition Activity by $16 S$ rRNA Gene Sequence. A total of 31 strains with high inhibition activity were analysed based on their 16S rRNA gene sequence. The strains were classified into 13 genera: Marinobacter sp., Pseudomonas sp., Alteromonas sp., Idiomarina sp., Halomonas sp., Alcanivorax sp., Thalassospira sp., Jannaschia sp., Stakelama sp., Erythrobacter sp., Chengkuizengella sp., Bacillus sp., and Microbacterium sp. (Figure 8). 


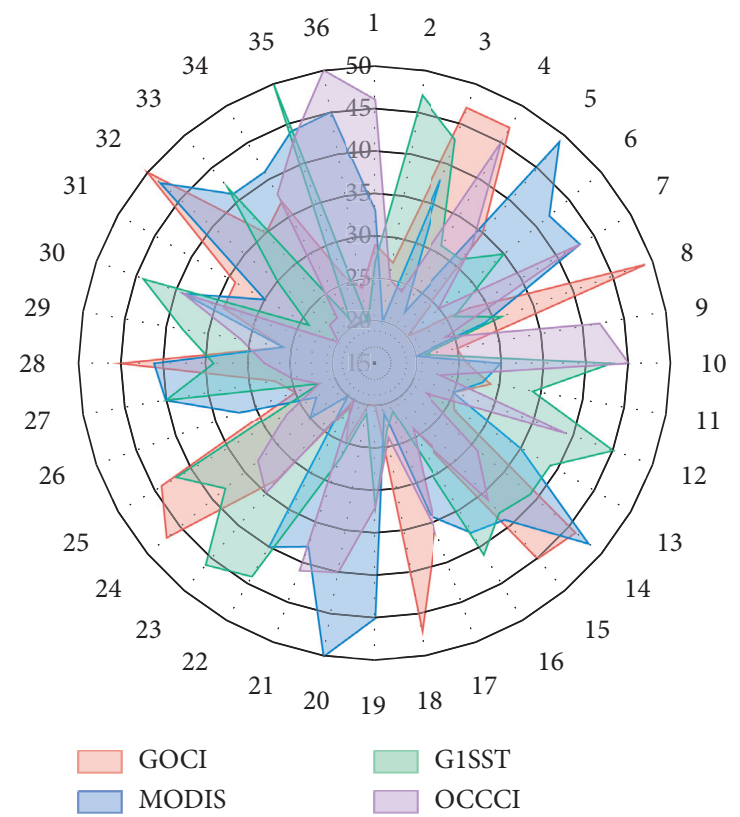

FIGURE 7: Variation of chlorophyll concentration at different periods.

TABLE 3: Fermentation medium, aflatoxin inhibition ratio, sampling station, and sampling depth for each strain.

\begin{tabular}{|c|c|c|c|c|}
\hline Strain name & Fermentation medium & Aflatoxin inhibition ratio (\%) & Sampling station & Sampling depth $(\mathrm{m})$ \\
\hline M57 & $\mathrm{R} 2 \mathrm{~A}$ & 93.54 & DY26III-S024-CTD06 & -1000 \\
\hline M58 & $\mathrm{R} 2 \mathrm{~A}$ & 96.01 & DY26III-S024-CTD06 & -1000 \\
\hline R34 & $\mathrm{R} 2 \mathrm{~A}$ & 97.15 & DY26III-S024-CTD06 & -150 \\
\hline $\mathrm{R} 104$ & $\mathrm{R} 2 \mathrm{~A}$ & 96.20 & DY26III-S034-CTD10 & -2600 \\
\hline MA16H & Starch casein & 100 & DY26III-TVG15 & -1523 \\
\hline $1-12 \mathrm{E}$ & $2216 \mathrm{E}$ & 90.63 & DY26III-S010-TVG01 & -3203 \\
\hline $1-40-5 R$ & $\mathrm{R} 2 \mathrm{~A}$ & 100 & DY26III-S010-TVG01 & -3203 \\
\hline $16-40-7 \mathrm{M}$ & M2 & 86.45 & DY26III-S042-TVG16 & -1598 \\
\hline $16-40-5 \mathrm{M}$ & M2 & 99.17 & DY26III-S042-TVG16 & -1598 \\
\hline $6-40-6 \mathrm{M}$ & M2 & 87.11 & DY26III-S016-TVG06 & -3059 \\
\hline $3-40-9 M$ & M2 & 100 & DY26III-S012-TVG03 & -3142 \\
\hline $15-8 \mathrm{M}$ & M2 & 100 & DY26III-TVG15 & -1523 \\
\hline $6-8 \mathrm{M}$ & M2 & 100 & DY26III-S016-TVG06 & -3059 \\
\hline $9-11 \mathrm{M}$ & M2 & 100 & DY26III-S020-TVG09 & -3073 \\
\hline $1-23 \mathrm{M}$ & M2 & 100 & DY26III-S010-TVG01 & -3203 \\
\hline $15-40-4 \mathrm{M}$ & M2 & 83.14 & DY26III-TVG15 & -1523 \\
\hline $16-40-3 \mathrm{M}$ & M2 & 100 & DY26III-S042-TVG16 & -1598 \\
\hline $16-14 \mathrm{M}$ & M2 & 97.34 & DY26III-S042-TVG16 & -1598 \\
\hline $21-12 \mathrm{M}$ & M2 & 100 & DY26III-S052-TVG21 & -451 \\
\hline $15-40-1 \mathrm{M}$ & M2 & 99.17 & DY26III-TVG15 & -1523 \\
\hline $16-40-1 \mathrm{M}$ & M2 & 82.06 & DY26III-S042-TVG16 & -1598 \\
\hline $4-2 \mathrm{G}$ & $\mathrm{G}$ & 100 & DY26III-S014-TVG04 & -3149 \\
\hline $16-5 \mathrm{G}$ & G & 92.07 & DY26III-S042-TVG16 & -1598 \\
\hline $15-1 \mathrm{~F}$ & $\mathrm{~F}$ & 82.39 & DY26III-TVG15 & -1523 \\
\hline $1-40-3 \mathrm{~F}$ & $\mathrm{~F}$ & 100 & DY26III-S010-TVG01 & -3203 \\
\hline $1 \mathrm{HAK}-4$ & Starch casein & 94.95 & DY26III-S010-TVG01 & -3203 \\
\hline FE7 & Gause No.1 & 84.36 & DY26III-S015-TVG05 & -3125 \\
\hline FE8 & Gause No.1 & 86.49 & DY26III-S015-TVG05 & -3125 \\
\hline FA13 & W1 & 100 & DY26III-S010-TVG01 & -3203 \\
\hline $15-2 \mathrm{I}$ & Glucose-asparagine & 94.62 & DY26III-TVG15 & -1523 \\
\hline $21 \mathrm{HAK}-6$ & $\mathrm{M} 2$ & 84.84 & DY26III-S052-TVG21 & -451 \\
\hline
\end{tabular}


strains exhibited significant inhibition against Aspergillus

TABLe 4: Fermentation medium, mycelial growth inhibition ratio, sampling station, and sampling depth for each strain.

\begin{tabular}{lcccc}
\hline Strain name & Fermentation medium & Mycelial growth inhibition ratio (\%) & Sampling station & Sampling depth (m) \\
\hline MA16H & Starch casein & $100 \%$ & DY26III-TVG15 & -1523 \\
$1-40-5 R$ & R2A & 94.2 & DY26III-S010-TVG01 & -3203 \\
$3-40-9 M$ & M2 & 93.85 & DY26III-S012-TVG03 & -3142 \\
$15-8 M$ & M2 & 94.61 & DY26III-TVG15 & -1523 \\
$9-11 \mathrm{M}$ & M2 & 97.60 & DY26III-S020-TVG09 & -3073 \\
$1-23 \mathrm{M}$ & M2 & 97.40 & DY26III-S010-TVG01 & -3203 \\
$16-40-3 \mathrm{M}$ & M2 & 86.84 & DY26III-S042-TVG16 & -1598 \\
$21-12 \mathrm{M}$ & M2 & 85.92 & DY26III-S052-TVG21 & -451 \\
FA13 & W1 & 100 & DY26III-S010-TVG01 & -3203 \\
$15-40-1 \mathrm{M}$ & M2 & 90.83 & DY26III-TVG15 & -1523 \\
$1-40-3 \mathrm{~F}$ & F & 99.21 & DY26III-S010-TVG01 & -3203 \\
\hline
\end{tabular}

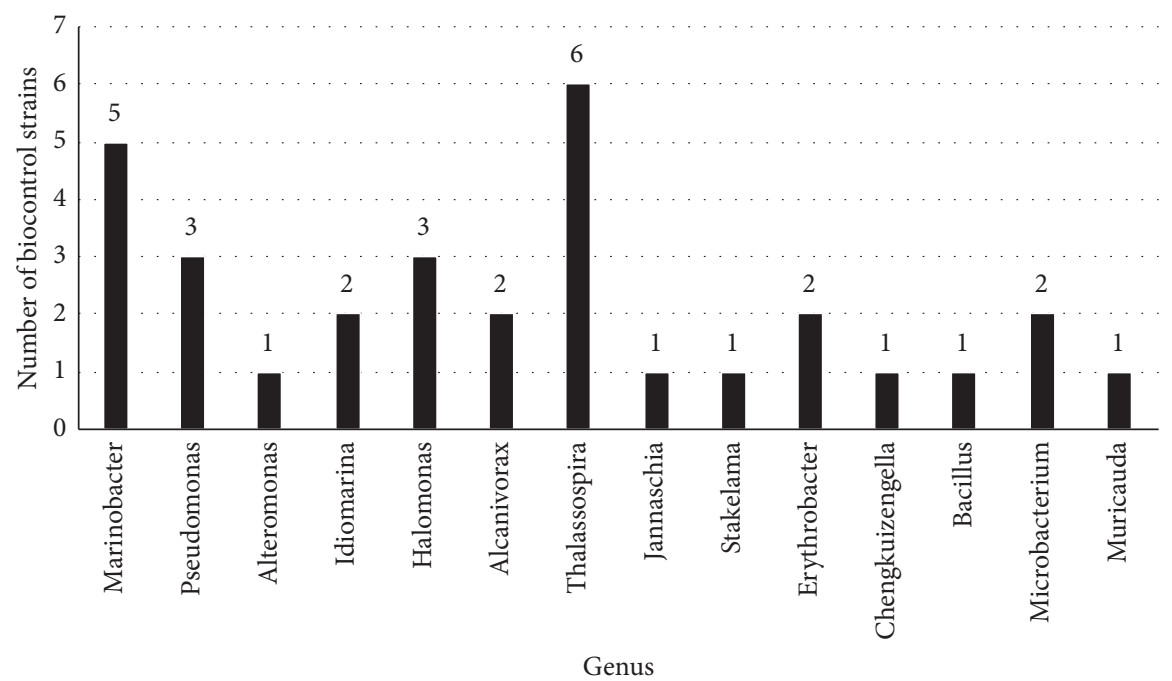

FIGURE 8: The strain number of different genera.

The phylogenetic tree in Figure 9 showed the diversity of strains with significant antagonism against aflatoxin. The 31 strains with antifungal and antiaflatoxigenic activity were dominated by populations of strains belonging to the genus Thalassospira (19.35\%) (Figure 1), including 6 strains, 1-405R, 15-40-4M, 16-40-1M, 16-40-3M, 16-40-5M, and 16-5G (Figure 2). The $16 \mathrm{~S}$ rRNA gene of these strains was $99.75 \%$ to 99.9\% identical to that of Thalassospira permensis. The second most common group of strains identified in the present study was from the genus Marinobacter. In total, 5 parasiticus mutant strain NFRI-95, accounting for $16.13 \%$. They were $16-40-7 \mathrm{M}, 4-2 \mathrm{G}, 6-40-6 \mathrm{M}, 6-8 \mathrm{M}$, and $15-8 \mathrm{M}$. These strains were $98.58 \%$ to $99.69 \%$ identical to Marinobacter algicola, Marinobacter adhaerens, and Marinobacter vinifirmus (Figure 9).

The numbers on the tree indicate the bootstrap percentage based on 1,000 replications and are shown for branches with more than $50 \%$ support. The scale bar represents 0.05 nucleotide substitutions per sequence position. 


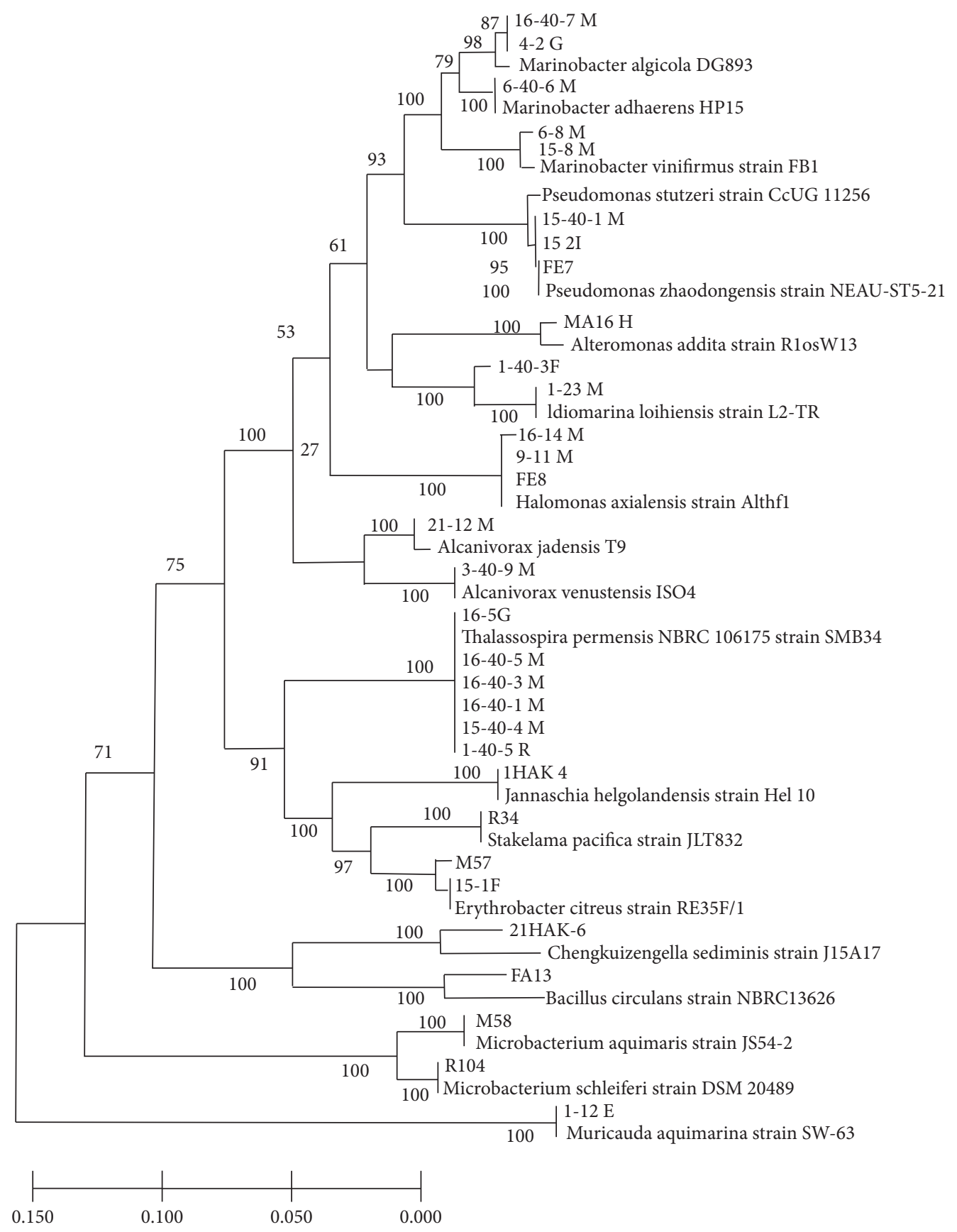

FIGURE 9: Neighbour-joining tree based on the 16S rRNA sequences of strains with high inhibition activity.

\section{Conclusion}

With the continuous development of GIS function, its application in various fields is increasing, and the application of GIS in the ocean is also booming. At the same time, with the support of government departments, the development of the marine economy is getting increased attention, and the rational development and utilization of marine resources and effective protection of the marine ecological environment are also gradually being paid attention to. The combination of RS and GIS technology can quickly and accurately realize the extraction of wetland information [21]. As the South Atlantic Ocean is a special ecosystem between land and sea, the traditional field investigation is not only time-consuming, laborious, small in scope, and influenced by human factors but also destructive to the ecosystem. The combination of RS and GIS technology not only overcomes the above-mentioned shortcomings and quickly and accurately realizes the extraction of wetland landscape distribution information but also can use the spatial analysis function of GIS to derive the transfer situation among wetland landscapes, providing a scientific basis for the protection and rational use of wetland resources. Given the characteristics of water 
resources in the Haile River Basin, a dynamic simulation model of ecological water use driven by multisource remote sensing data was constructed using remote sensing technology and coupled with a hydrological model, and an ecohydrological database was built. This paper takes the South Atlantic Ocean as the study and has achieved some results in exploring the marine ecological environment using GIS technology, but there are some shortcomings in the study due to the limitations in data, technical means, and personal knowledge reserve. We expect that we can use better evaluation methods and improve the shortcomings in the future to study the ecological environment of other important islands and provide a reference basis for the study of the natural and socioeconomic development of China's islands. Future research in this area can also be explored to a deeper and wider dimension. It is believed that, with the continuous development of marine GIS, the marine environment analysis and evaluation of early warning business is more than an efficient business system; under the general trend of the smart ocean, it will develop to a higher level of wisdom based on GIS.

In this study, thirty-two seawater and sediment samples were collected from different depths within the scope of $5 \mathrm{~m}$ to $3203 \mathrm{~m}$ at 18 sampling sites (CTD06, CTD10, CTD11, TVG1, TVG2, TVG3, TVG4, TVG5, TVG6, TVG8, TVG9, TVG10, TVG12, TVG13, TVG14, TVG15, TVG16, and TVG21) in the South Atlantic Ocean. 1017 culturable strains from deep seawater and sediments in South Atlantic Ocean were isolated and identified. A total of 31 deep-sea strains were confirmed to have significant antagonism against aflatoxin production with an inhibition ratio exceeding $80 \%$, including 11 strains with a mycelial growth inhibition ratio exceeding $80 \%$ simultaneously. These 31 strains clustered into 13 genera: Marinobacter sp., Pseudomonas sp., Alteromonas sp., Idiomarina sp., Halomonas sp., Alcanivorax sp., Thalassospira sp., Jannaschia sp., Stakelama sp., Erythrobacter sp., Chengkuizengella sp., Bacillus sp., and Microbacterium sp., which were dominated by populations of strains belonging to the genus Thalassospira (19.35\%). Strains with both antifungal activity and antiaflatoxigenic activity were the most fermented by the M2 medium. The strains from TVG15 were capable of not only inhibiting aflatoxin production but also inhibiting mycelial growth of Aspergillus parasiticus mutant strain NFRI-95.

\section{Data Availability}

The data used to support the findings of this study are available from the corresponding author upon request.

\section{Conflicts of Interest}

The authors declare that they have no conflicts of interest.

\section{Acknowledgments}

This research work was supported by COMRA Project (DY125-15-R-01 and DY135-B2-17).

\section{References}

[1] R. Wang, M. Xu, and Y. Ding, “The development of marine oil spill operational system based on GIS," Journal of Software, vol. 9, no. 8, pp. 2050-2055, 2014.

[2] L. I. Dan, W. U. Baosheng, and C. Bowei, "Review of water body information extraction based on satellite remote sensing," Journal of Tsinghua University, vol. 60, no. 2, pp. 147$161,2020$.

[3] D. S. Boyd and G. M. Foody, "An overview of recent remote sensing and GIS based research in ecological informatics," Ecological Informatics, vol. 6, no. 1, pp. 25-36, 2011.

[4] T. Blaschke, "Object based image analysis for remote sensing," ISPRS Journal of Photogrammetry and Remote Sensing, vol. 65, no. 1, pp. 2-16, 2010.

[5] T. Pei, J. Xu, Y. Liu et al., "GIScience and remote sensing in natural resource and environmental research: status quo and future perspectives," Geography and Sustainability, vol. 2, no. 3, pp. 207-215, 2021.

[6] Z. Münch and J. Conrad, "Remote sensing and GIS based determination of groundwater dependent ecosystems in the Western Cape, South Africa," Hydrogeology Journal, vol. 15, no. 1, pp. 19-28, 2007.

[7] M. S. Boori, V. Voženílek, and K. Choudhary, "Land use/cover disturbance due to tourism in Jeseníky Mountain, Czech Republic: a remote sensing and GIS based approach," The Egyptian Journal of Remote Sensing and Space Science, vol. 18, no. 1, pp. 17-26, 2015.

[8] K. Choudhary, M. S. Boori, and A. Kupriyanov, "Spatial modelling for natural and environmental vulnerability through remote sensing and GIS in Astrakhan, Russia," The Egyptian Journal of Remote Sensing and Space Science, vol. 21, no. 2, pp. 139-147, 2018.

[9] Y. Twumasi and E. Merem, "GIS and remote sensing applications in the assessment of change within a coastal environment in the Niger delta region of Nigeria," International Journal of Environmental Research and Public Health, vol. 3, no. 1, pp. 98-106, 2006.

[10] J. M. Mironga, "Geographic information systems (gis) and remote sensing in the management of shallow tropical lakes," Applied Ecology and Environmental Research, vol. 2, no. 1, pp. 83-103, 2004.

[11] A. Y. Ivanov and V. V. Zatyagalova, "A GIS approach to mapping oil spills in a marine environment," International Journal of Remote Sensing, vol. 29, no. 21, pp. 6297-6313, 2008.

[12] M. Yasir, S. Hui, H. Binghu, and S. U. Rahman, "Coastline extraction and land use change analysis using remote sensing (RS) and geographic information system (GIS) technology - a review of the literature," Reviews on Environmental Health, vol. 35, no. 4, pp. 453-460, 2020.

[13] L. Z. Yu, Y. Xu, and S. M. Cai, "A GIS-based study on recent environmental change in Honghu Lake," Journal of Lake Sciences, vol. 5, no. 4, pp. 350-357, 1993.

[14] I. N. Radiarta, S. I. Saitoh, and A. Miyazono, "GIS-based multi-criteria evaluation models for identifying suitable sites for Japanese scallop (Mizuhopecten yessoensis) aquaculture in Funka Bay, southwestern Hokkaido, Japan,” Aquaculture, vol. 284, no. 1-4, pp. 127-135, 2008.

[15] D. Song, Z. Gao, and H. Zhang, "GIS-based health assessment of the marine ecosystem in Laizhou Bay, China," Marine Pollution Bulletin, vol. 125, no. 1-2, pp. 242-249, 2017.

[16] T. T. Werner, A. Bebbington, and G. Gregory, "Assessing impacts of mining: recent contributions from GIS and remote 
sensing," The Extractive Industries and Society, vol. 6, no. 3, pp. 993-1012, 2019.

[17] Z. Qingbo, "Status and tendency for development in remote sensing of agriculture situation," Journal of China agricultural resources and regional planning, vol. 25, no. 5, pp. 9-14, 2004.

[18] W. Kai, Y. Peisheng, M. Rui, J. Wenwen, and S. Zongze, "Diversity of culturable bacteria in deep-sea water from the south Atlantic ocean," Bioengineered, vol. 8, no. 15, pp. 572-584, 2017.

[19] B. Hoersch, G. Braun, and U. Schmidt, "Relation between landform and vegetation in alpine regions of Wallis, Switzerland. A multiscale remote sensing and GIS approach," Computers, Environment and Urban Systems, vol. 26, no. 2-3, pp. 113-139, 2002.

[20] G. Liu, L. Zhang, Q. Zhang, Z. Musyimi, and Q. Jiang, "Spatiotemporal dynamics of wetland landscape patterns based on remote sensing in yellow river delta, China," Wetlands, vol. 34, no. 4, pp. 787-801, 2014.

[21] J.-K. Choi, H.-J. Oh, B. J. Koo, J.-H. Ryu, and S. Lee, "Crustacean habitat potential mapping in a tidal flat using remote sensing and GIS," Ecological Modelling, vol. 222, no. 8, pp. 1522-1533, 2011. 